\title{
Zum Zusammenhang von Wohnortswahl und Schulqualität: theoretische Überlegungen und empirische Befunde
}

\section{Maja Coradi Vellacott}

Die Zusammensetzung der Schülerschaft beeinflusst die individuellen Leistungen der Neuntklässlerinnen und Neuntklässler in der Schweiz - das haben Analysen der PISA-Daten 2000 und 2003 gezeigt. Es stellt sich die Frage, warum eine Konzentration von Schülerinnen und Schülern mit sprachlichen und sozio-ökonomischen Benachteiligungen in bestimmten Schulhäusern nicht einfacher verhindert werden kann. Einen Einblick in den Prozess der sozialen Segregation von Gemeinden und Wohnquartieren bieten Theorien der sozial-räumlichen Differenzierung und der Wohnortswahl. Vor diesem theoretischen Hintergrund wird der Zusammenhang von Gemeindemerkmalen, wie zum Beispiel der Steuerbelastung oder der Wohnatmosphäre, mit dem Wohnort von gutsituierten Personen deskriptiv untersucht. Ebenfalls gezeigt wird in diesem Beitrag der Einfluss des Reichtums von Gemeinden auf die Ausstattung ihrer Schulhäuser. Die theoretischen und empirischen Erkenntnisse machen deutlich, dass die Wohnortswahl statushoher Personen im Zusammenhang steht mit der sozialen Entmischung der Schülerschaft in Schweizer Schulhäusern. Mehrfachbelastungen ergeben sich dadurch vor allem für fremdsprachige, sozial benachteiligte Jugendliche, welche nicht in reichen Gemeinden wohnen.

\section{Einleitung und Fragestellung}

Analysen der PISA-Daten 2000 und 2003 zeigen, dass sich die durchschnittlichen Leistungen der Neuntklässlerinnen und Neuntklässler in der Schweiz substanziell zwischen den Schulen (auch des gleichen Anspruchsniveaus) unterscheiden (z.B. Coradi Vellacott, Hollenweger, Nicolet \& Wolter, 2003; Ramseier $\&$ Brühwiler, 2003; Moser \& Berweger, 2004). Diese Unterschiede sind unter anderem auf die Zusammensetzung der Schülerschaft zurückzuführen. Auch Effekte der Schülerzusammensetzung auf individuelle Leistungen sind bereits in verschiedenen Studien dokumentiert worden - in der Schweiz (ebenda) wie auch im Ausland (Deutschland (PISA): z.B. Schümer, 2004; Deutschland (andere Stufen): Ditton \& Krüsken, 2006; angelsächsischer Raum (Überblick): 
Thrupp, Lauder \& Robinson, 2002). Uneinheitlich sind die Befunde, wenn es um die Frage geht, welche der Merkmale der Schülerzusammensetzung (soziale, ethnische, sprachliche, leistungsmässige Zusammensetzung) die individuelle Leistung am stärksten beeinflussen. Allerdings sind diese verschiedenen Aspekte der Schülerzusammensetzung so stark konfundiert, dass sich ihre Effekte nur schwer voneinander trennen lassen (Stanat, 2006).

In der Schweiz ist festgestellt worden, dass die individuellen Leseleistungen in Schulhäusern mit einem hohen Anteil an fremdsprachigen Schülerinnen und Schülern niedriger ausfallen (unter Kontrolle der sozio-ökonomischen Zusammensetzung der Schülerschaft, des Schultyps sowie individuellen Merkmalen der familiären Herkunft). Fremdsprachige und sozio-ökonomisch benachteiligte Schülerinnen und Schüler sind in besonderem Masse betroffen von diesem Umstand. Sie erfahren einerseits eine zusätzliche Benachteiligung, neben derjenigen durch ihren familiären Hintergrund, wenn sie in einem Schulhaus unterrichtet werden, in dem mehr als 40 Prozent der Schülerschaft fremdsprachig ist (Coradi Vellacott et al., 2003; für den Kanton Zürich: Moser und Rhyn, 1997, 2000). Gleichzeitig gehören sie andererseits zu denen, die am meisten profitieren können von einem relativ höheren durchschnittlichen sozio-ökonomischen Status (Coradi Vellacott, in Vorb.).

Es stellt sich nun die Frage, warum eine Ansammlung von 40 Prozent und mehr fremdsprachigen und sozial benachteiligten Schülerinnen und Schülern in bestimmten Schulhäusern nicht einfacher verhindert werden kann, zumal der Anteil von Ausländerinnen und Ausländern in der Schweiz rund 21 Prozent beträgt. Da in der Schweiz die Schule nicht frei gewählt werden kann, muss davon ausgegangen werden, dass eine Konzentration von Jugendlichen aus bestimmten familiären Verhältnissen in einem Schulhaus mit einer geographischen Überrepräsentation dieser Jugendlichen im Umfeld der Schule - und damit mit der Wohnortswahl - zu tun hat. Das Ziel dieses Artikels ist es, den Prozess der sozialräumlichen Differenzierung in der Schweiz theoretisch und empirisch näher zu beleuchten und seine Auswirkungen auf die Lernvoraussetzungen in den Schulen zur Debatte zu stellen. Nach einer kurzen theoretischen Annäherung an den Begriff der sozial-räumlichen Differenzierung werden mögliche Zusammenhänge von Wohnortswahl und Schulqualität unter Einbezug von Theorie und empirischen Forschungsresultaten erörtert. Danach werden zwei Fragestellungen anhand eines erweiterten PISA-Datensatzes analysiert. Erstens wird untersucht, ob auf der Ebene der Gemeinden der Schweiz eine sozial-räumliche Segregation zu beobachten ist. Zweitens wird es darum gehen, den Einfluss des Reichtums von Gemeinden auf bestimmte schulische Lernvoraussetzungen zu überprüfen. 


\section{Konstituierung von Räumen: Wohnortswahl}

Für eine Untersuchung der Verteilung bestimmter Bevölkerungsgruppen in einem geographischen Raum ist eine breite theoretische Basis vorhanden. Insbesondere in der soziologischen Theorie wurde die Verknüpfung von räumlicher Differenzierung und sozialer Differenzierung mehrfach thematisiert (Harvey, 1973; Castells, 1976; Friedrichs, 1977; Bourdieu, 1985, 1991; Giddens, 1987; Löw, 2001). Die folgenden Untersuchungen der sozial-räumlichen Segregation in der Schweiz basieren auf Bourdieus Ansatz. Anders als Giddens oder die frühe Stadt- und Raumsoziologie geht Bourdieu davon aus, dass Raum nicht nur eine Bedingung für menschliches Handeln darstellt, sondern selbst sozial konstruiert ist. Nach seinem Verständnis ist insbesondere die Hierarchisierung in der sozialen und räumlichen Differenzierung wesentlich. Damit ist angesprochen, dass die Möglichkeit, Räume zu konstituieren und räumliche Anordnungen durchzusetzen, nicht für alle Gesellschaftsmitglieder gleich ist (Löw, 2001). Das heisst zum Beispiel ganz konkret, dass nicht alle Personen beliebig bestimmen können, wo sie wohnen. Eine Familie mit hohem Einkommen kann sich hohe Miet- und Bodenpreise leisten. Benachteiligte und insbesondere sozialleistungsabhängige Familien hingegen sind an den Kämpfen um Wohnorte oder «Territorien» gemeinhin nicht beteiligt (Dear \& Wolch, 1991). Viele müssen entweder in die billigsten verfügbaren Wohnungen ziehen oder bekommen eine Sozialwohnung zugeteilt. Kommunale und baugenossenschaftliche Wohnungen sind nun in der Schweiz aber nicht gleichmässig auf verschiedene Gemeinden verteilt gebaut worden, sondern es existiert eine grosse Verteilungsdisparität (Arend, 2004). Auch ist der Zugang zum Wohnungsmarkt für sozio-ökonomisch benachteiligte Personen oft erschwert. Die Ursache davon liegt am häufigsten in der Diskriminierung bestimmter Nachfragegruppen durch die Vermieter und/oder Mitbewohner (Arend, Kellerhals Spitz \& Mächler, 1990).

Den sozio-ökonomisch benachteiligten Familien wird also der Zugang zu bestimmten Quartieren oder Gemeinden über den Immobilienpreis sowie über andere Ausschlussmechanismen in Wohnungs- und Sozialpolitik verwehrt. Bourdieu spricht in diesem Zusammenhang von einem Klub-Effekt und einem Ghetto-Effekt und beschreibt die Ghettobewohner als eine «im Nichtbesitz homogene Population» (Bourdieu, 1991, S.32). Diese Beschreibung passt auf schweizerische Verhältnisse: In der Schweiz sind selten ethnisch homogene Stadtteile oder Gemeinden zu finden. ${ }^{1}$ Stattdessen sind Ansammlungen von in verschiedener Hinsicht Benachteiligten zu beobachten. Dazu gehören in der Schweiz sowohl die sozio-ökonomisch benachteiligten wie auch die meisten der immigrierten Familien. ${ }^{2}$

Um den Prozess der sozial-räumlichen Entmischung differenziert abzubilden und damit einer Analyse zugänglich zu machen, muss jedoch auch die Frage geklärt werden, warum sich privilegierte Eltern überhaupt hohe Mietpreise leisten. Es ist anzunehmen, dass auch sozio-ökonomisch gutgestellte Eltern ihre 
Wohnkosten gering halten möchten. Die theoretische Antwort ist bereits angetönt worden. Miet- und Immobilienpreise sind nicht ausschliesslich monetär zu sehen, sondern haben auch Bedeutung als Instrument des sozialen Ausschlusses. Durch Ausschluss von sozial benachteiligten und fremdsprachigen Familien zum Beispiel entstehen sozial homogene Nachbarschaften und Gemeinden - auch Schulgemeinden. Immobilienpreise können deshalb als Indikatoren für zahlreiche weitere Merkmale einer Wohnumgebung benutzt werden. Um welche Merkmale es sich dabei handelt und welche Dynamik ihre Beziehungen untereinander annehmen kann, wird im nächsten Kapitel beschrieben.

\section{Wohnortswahl und Schulqualität}

Dass die Schule bei einem Umzug ein wichtiges Kriterium sein kann, hat Tiebout bereits 1956 in einem Aufsatz festgestellt. Er hat dargelegt, dass die Wahl des Wohnorts vom Arbeitsort, von den Steuern, aber auch von der Verfügbarkeit öffentlicher Güter abhänge. Kesselring (1979) nennt spezifisch für die Schweiz eine tiefe Steuerbelastung, eine gute Ausstattung mit Infrastrukturanlagen (Einkaufsmöglichkeiten, Schulen, usw.), die Agglomerationsnähe sowie eine sonnige und aussichtsreiche Lage als Bedingungen für den sogenannten Wanderungsentscheid von Haushalten. Dass nicht nur die Verfügbarkeit, sondern auch Annahmen über die Qualität einer Schule umzugswillige Eltern in ihrem Entscheid beeinflussen, ist insbesondere in amerikanischen Studien betont worden (siehe z.B. Bayer, Ferreira \& McMillan, 2004). Da in der Schweiz aber kein sogenanntes Schulranking betrieben wird, ist diese Qualität für Eltern hierzulande schwer abschätzbar. In der Regel werden Signale benutzt, um die Qualität einer Schule zu beurteilen. Als Signale können die Zusammensetzung der Schülerschaft, die Qualität der Lehrpersonen oder die Ausstattung des Schulhauses fungieren. Auch diese Informationen sind insbesondere Eltern, welche beim Umzug die Gemeinde wechseln, jedoch nicht unbedingt bekannt. Es wird im Folgenden deshalb postuliert, dass letztlich die Immobilienpreise zur Beurteilung der Schulqualität herangezogen werden. Es wird dargelegt, dass Immobilienpreise zum Beispiel als Indikator für die "Qualität» der zukünftigen Schulkameradinnen und -kameraden (peers) der eigenen Kinder oder für die Ausstattung des Schulhauses genutzt werden.

Wohnen, wo die Kinder aus gutem Hause zur Schule gehen Verschiedene Studien aus Ländern, in denen freie Schulwahl möglich ist, haben festgestellt, dass die zukünftigen peers der eigenen Kinder für Eltern bei der Schulwahl wichtig sind (OECD,1994). Zahlreiche Forschungsergebnisse aus den USA weisen auch tatsächlich darauf hin, dass dem Wohlstand von Nachbarschaft und Gemeinde eine grosse Bedeutung für den Bildungserfolg zukommt (z.B. Sun, 1999; Leventhal \& Brooks-Gunn, 2000; Willms, 2002). Die Überle- 
gung, mit welchen peers das eigene Kind zur Schule gehen und nachbarschaftliche Kontakte haben wird, ist für umzugswillige Eltern also unter Umständen durchaus im Sinne der Maximierung des eigenen Nutzens.

Eltern möchten, dass ihre Kinder von diesen peers möglichst leistungsförderlich beeinflusst werden. Die "Qualität» der Schülerschaft in der Gemeinde wird deshalb anhand von deren Fähigkeiten und derem Verhalten beurteilt. Solche Informationen sind aber schwer beobachtbar. Um sie dennoch zu erhalten, wird auf die Eltern der zukünftigen Schulkameradinnen und -kameraden zurückgegriffen - es wird angenommen, dass gut ausgebildete, sozio-ökonomisch privilegierte Eltern fähige und geförderte Kinder haben. Eltern möchten also dorthin ziehen, wo die Kinder gutsituierter Eltern wohnen. Eine Studie aus den USA, wo die Eltern auch direkte Informationen über die Qualität der Schulen erhalten, zeigt, dass tatsächlich ein grosser Teil des Einflusses der Schulqualität auf die Wohnortswahl indirekt über die Präferenz bestimmter Nachbarschaften wirkt (Bayer, Ferreira \& McMillan, 2004). Umzugswillige Eltern müssen also Merkmale eines Wohnorts beziehungsweise einer Gemeinde finden, die als Signale für eine Selbstselektion statushoher Eltern in die gleichen Gemeinden dienen können. Um solche Signale zu finden, reicht es nun nicht Präferenzen aufzulisten, die statushohe Eltern bei der Wohnortswahl haben. Denn sozio-ökonomisch benachteiligte und fremdsprachige Eltern haben nicht zwingend andere Wünsche an ihren Wohnort als statushohe (auch wenn der Nutzen der einzelnen Präferenzen unter Umständen anders gewichtet wird). Es müsste gleichzeitig nach Gemeinden gesucht werden, die von statusniedrigen, einkommensschwachen Eltern nicht gewählt werden können.

Wie Bourdieu (1991) feststellt, ermöglicht es erst ökonomisches, soziales und kulturelles Kapital, sich unerwünschte Personen und Dinge vom Leib zu halten sowie sich den begehrten Personen und Dingen zu nähern. Personen ohne Kapital können physisch von den sozial als selten eingestuften Gütern ferngehalten und dazu gezwungen werden, mit den unerwünschtesten Personen und am wenigsten seltenen Gütern zu verkehren. Aufgrund der oben beschriebenen Entscheidungsdynamik sind es deshalb in erster Linie diejenigen Gemeinden, in denen die Immobilien- und Mietpreise sehr hoch sind, die als Wohn- und Schulort von privilegierten Eltern priorisiert werden. In solchen Gemeinden zu wohnen, können sich sozio-ökonomisch benachteiligte Familien nicht leisten.

Es wird hier also postuliert, dass Eltern die Immobilienpreise in einer Gemeinde als ein Signal betrachten, das viele zusätzliche Informationen in sich trägt, eben auch die Information über zukünftige peers der eigenen Kinder. Stellvertretend für die Immobilienpreise kann auch die Steuerattraktivität als Signal betrachtet werden, da die beiden Merkmale eng zusammenhängen (z. B. Schmidheiny, 2002). Der sozio-ökonomische Status der Eltern wird also anhand der Immobilienpreise oder der Steuerbelastung in deren Wohngemeinde "geschätzt».

Als Fazit dieser Herleitung kann festgehalten werden: Eltern, welche sich für eine steuerattraktive Wohngemeinde entscheiden, stellen auch gleich sicher, dass ihre 
Kinder mit sozio-ökonomisch privilegierten peers zur Schule gehen werden. $\mathrm{Ob}$ dieses Fazit auch empirisch untermauert werden kann, soll weiter unten anhand eines erweiterten PISA-Datensatzes geprüft werden.

\section{Wohnen, wo die Schulhäuser gut ausgestattet sind}

Als weiteres Signal für die Qualität einer Schule ist oben die Qualität der Ausstattung genannt worden. Mit Ausstattung ist die Versorgung der Schule mit pädagogischem Lehrmaterial (dazu gehören Bibliotheksbücher, Laborutensilien oder Computer), oder die Einrichtung gemeint. Ob die Ausstattung einer Schule die Qualität des Unterrichts oder die Leistungen der Schülerinnen und Schüler massgeblich beeinflusst, kann an dieser Stelle nicht schlüssig beurteilt werden. Es muss jedoch davon ausgegangen werden, dass neben der Verfügbarkeit von Lehrmaterial oder Einrichtungen auch deren Nutzung überprüft werden müsste, um den Zusammenhang adäquat abzubilden. Analysen der PISAStudie 2003, welche die Nutzung von Informations- und Kommunikationstechnologien in der Schule auf einen Zusammenhang mit den Leistungen hin überprüft haben, haben allerdings keine signifikanten Effekte gefunden (Ramseier \& Holzer, 2005).

Trotzdem scheint es für Eltern wichtig zu sein, wie die Schule ihrer Kinder ausgerüstet ist. In Studien aus anderen Ländern (z.B. Schweden, Grossbritannien) wird die Ausstattung als ein Kriterium genannt, dem die Eltern bei der Wahl der Schule Gewicht beimessen (OECD, 1994). Es ist also durchaus vorstellbar, dass auch Eltern in der Schweiz, welche Schulwahl über die Wohnortswahl betreiben, besser ausgestattete Schulhäuser vorziehen. Es stellt sich hier aber die Frage, ob eine Schweizer Gemeinde überhaupt ein besser ausgestattetes Schulhaus als eine andere haben kann, oder ob dies durch den Finanzausgleich zwischen reichen und armen Gemeinden verhindert wird. Werden die Finanzierungsprozesse näher betrachtet wird klar, dass es den Gemeinden frei steht, Ausgaben für die Schule zu tätigen, welche über die vorgeschriebene Ausstattung eines Schulhauses hinausgehen (z.B. zusätzliche Computer für Schülerinnen und Schüler). Solche Ausgaben werden meist in der Gemeindeversammlung oder von Schulkommissionen der Gemeinden beschlossen. ${ }^{3}$ Sie sind daher abhängig von den vorhandenen finanziellen Mitteln, welche wiederum eng mit der Steuerkraft der Einwohner zusammenhängen.

Die Frage, ob qualitative Unterschiede in der Ausstattung der Schulhäuser verschiedener Gemeinden möglich sind, muss also bejaht werden. Es gibt auch bereits Hinweise darauf, dass solche Unterschiede tatsächlich bestehen. Schmidheiny (2002) zeigt für zwei städtische Schweizer Gemeinden auf, dass in einer Gemeinde mit hoher Steuerbelastung (also tendenziell tiefem Steuersubstrat) weniger Geld für öffentliche Güter ausgegeben wird als in einer steuerattraktiven Gemeinde.

Es wird hier postuliert, dass Eltern, die bei ihrer Wohnortswahl die Qualität der Schule berücksichtigen, anhand der Steuerbelastung in einer Gemeinde auch 
die Ausstattung der Schule beurteilen. Dies tun sie, indem sie annehmen, dass die Steuerbelastung in einer Gemeinde als Signal für den sozio-ökonomischen Status der dort ansässigen Eltern (beziehungsweise zukünftigen Eltern) betrachtet werden kann. Das Fazit dieser Herleitung lautet also: Eltern, welche sich für eine steuerattraktive Wohngemeinde entscheiden, stellen auch gleich sicher, dass ihre Kinder in einem gut ausgestatteten Schulhaus unterrichtet werden.

\section{Mode l L}

Neben der Steuerbelastung (und der darin enthaltenen Information über die Qualität der Schule) sind jedoch auch andere Faktoren wesentlich bei der Wohnortswahl. Die Nähe zum Arbeitsort wird zum Beispiel von verschiedenen Autoren als wichtiges Kriterium genannt (Tiebout, 1956; Kesselring, 1979). Auch darf ein weiterer Faktor bei der Wohnortswahl nicht unterschätzt werden, der jedoch nicht einfach zu erfassen ist: die Atmosphäre eines Ortes. Eine von statushohen Personen bewohnte Gemeinde vermittelt anderen Gutsituierten das Gefühl, heimisch zu sein (Löw, 2001). Dieses Gefühl mag sozio-ökonomisch privilegierten Personen unter anderem ebenfalls als Indikator für die Qualität der Schule dieser Gemeinde dienen, denn es kann, ähnlich wie die tiefe Steuerbelastung, für viele statushohe Schulkameradinnen und Schulkameraden des eigenen Kindes stehen. Die Nähe zum Arbeitsort und die Atmosphäre eines Ortes werden als Kontrollvariablen im Modell aufgeführt.

Andere Faktoren wie die Aussicht oder die Versorgung mit Infrastruktur werden nicht im Modell zu finden sein, da die Datengrundlage für eine Überprüfung ihres Zusammenhangs mit der Wohnortswahl nicht genügt. ${ }^{4}$

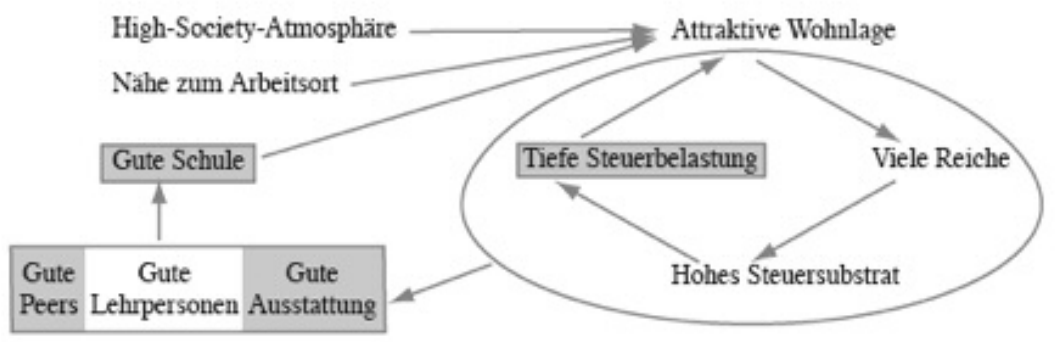

\section{Abbildung 1: Einflïsse auf die Wohnortswahl}

Der Prozess der sozial-räumlichen Entmischung kann als kumulativer Kreislauf verstanden werden, der mit jedem «Durchlauf» mehr sozial-räumliche Segregation und mehr Unterschiede zwischen den Schulen schafft. Denn aufgrund der oben beschriebenen Zusammenhänge ziehen Gemeinden mit gutsituierter Einwohnerschaft immer wieder neu statushohe Personen an, die ihren Kindern die besten schulischen Bedingungen garantieren möchten. 
$\mathrm{Ob}$ eine systematische sozial-räumliche Segregation auch in der Verteilung der Bevölkerung auf die Schweizer Wohngemeinden beobachtet werden kann, wird im Folgenden als erste Fragestellung überprüft. Da die verschiedenen Kapitalsorten gegenseitig aufeinander bezogen sind (soziales Kapital bezieht sich zum Beispiel stets auch auf das damit erreichbare ökonomische und kulturelle Kapital) wird hier angenommen, dass in den Profilen der Schweizer Gemeinden gewisse Muster auszumachen sind. Konkret wird untersucht, ob die Steuerbelastung einer Gemeinde, deren Wohnatmosphäre sowie deren Betriebsstruktur einen signifikanten Zusammenhang aufweisen mit der Anzahl an sozio-ökonomisch privilegierten Einwohnerinnen und Einwohner. Die Untersuchung dieser ersten Fragestellung muss als explorative Annäherung an die sozial-räumliche Differenzierung in der Schweiz verstanden werden, und dies aus folgenden Gründen: Es ist ein grosser Nachteil, dass die Analysen nur auf Gemeinde- und nicht auf Quartierebene durchgeführt werden können. Dadurch entziehen sich unter Umständen wesentliche Prozesse der sozial-räumlichen Segregation der Beobachtung. Weiter ist es durch die Querschnittsstruktur der Daten unmöglich zu beurteilen, ob die postulierten Zusammenhänge in eine bestimmte Richtung wirken (intrinsisches Endogenitätsproblem). Trotzdem kann von den Analysen jedoch ein Einblick in die Hintergründe von sozial-räumlich bedingten Unterschieden der Schulqualität erwartet werden.

Die zweite Fragestellung widmet sich den Lernvoraussetzungen im Schulhaus: es wird untersucht, ob der Reichtum (beziehungsweise die Steuerattraktivität) einer Gemeinde an sich für die materiellen Lernvoraussetzungen in einer Schule von Bedeutung ist, wie dies Eltern gemäss der beschriebenen theoretischen Überlegungen annehmen.

\section{Methode und Exploration der Daten}

\section{St i chproben}

Für die Ergänzung der PISA-Daten mit Informationen über politische Gemeinden wurden verschiedene Datenquellen benutzt. Der Grossteil der Informationen konnte aus den Daten der Volkszählung 2000 gewonnen werden. Weitere Angaben stammen aus der Betriebszählung 2001 oder wurden, im Falle der Steuerbelastung, teilweise selbst berechnet. Die Operationalisierungen der einzelnen Variablen werden in der Tabelle 1 beschrieben.

Die Stichprobe, mit der die erste Fragestellung analysiert wird, umfasst 148 Gemeinden (aus 19 Kantonen), welche sich im Einzugsgebiet von Schulen befinden, die sich an PISA 2000 beteiligt haben; also Gemeinden, die ihre Schülerinnen und Schüler im Jahr 2000 in eine Schule schickten, in der die PISA-Tests durchgeführt worden sind. Es konnten jedoch nicht alle dieser Schulen für den Vergleich von Standortvariablen beibehalten werden, da sie zum Teil Neuntklässlerinnen und Neuntklässler aus zu vielen verschiedenen Gemeinden unter- 
richten. ${ }^{5}$ Die Schulen mit sehr grossem Einzugsgebiet und damit auch die Gemeinden, in denen ihre Schülerinnen und Schüler wohnen, wurden für diese Analyse ausgeschlossen. Ebenfalls ausgeschlossen wurden kantonal geführte Schulen (z.B. Gymnasien), da sie einerseits Jugendliche aus sehr vielen Gemeinden zusammenziehen und andererseits finanziell von den Gemeinden unabhängig sind. Für die Untersuchungen der ersten Fragestellung werden im Folgenden ausschliesslich die Gemeindevariablen und damit die Stichprobe der $148 \mathrm{Ge}-$ meinden verwendet. Darunter sind auch Gemeinden, welche ihre Neuntklässlerinnen und Neuntklässler in ein und dieselbe Schule schicken. Zur Beantwortung der Forschungsfrage ist es jedoch nicht von Bedeutung, welche Schule diese Jugendlichen besuchen. Die Aussagen, welche im Rahmen der Beurteilung der Fragestellung gemacht werden, beziehen sich auf diejenigen 148 Gemeinden, die zum Einzugsgebiet der 114 Schulen gehören, welche für die Überprüfung der weiteren Fragestellung herangezogen werden können. In dieser Analyse steht die Untersuchung von möglichen Zusammenhängen im Vordergrund, und nicht die Beschreibung der Grundgesamtheit der schweizerischen Gemeinden. Da keine Gewichtung vorgenommen wurde, ist die Repräsentativität der Resultate für alle Schweizer Gemeinden nicht gewährleistet.

Für die zweite Fragestellung werden auch Schulvariablen benötigt. Die Stichprobe reduziert sich damit auf 114 Schulen; in den Fällen, in denen mehrere (aber nicht mehr als drei) Gemeinden zu einer Schule gehören, wurden gewichtete Mittelwerte für alle Gemeindevariablen berechnet. Dieser Datensatz weist gegenüber der ursprünglichen Schulstichprobe eine leichte Überrepräsentation von Schulen mit hohem Anteil an fremdsprachigen Jugendlichen auf. Dies kann zum Beispiel eine Folge der Reduzierung der Stichprobe um sämtliche Gymnasien sein. Als Konsequenz werden die Ergebnisse der Analysen nicht in generalisierender Form präsentiert.

\section{Variablen}

Tabelle 1 zeigt die Variablen, die zur Charakterisierung der sozial-räumlichen Differenzierung ausgewählt wurden.

\section{Erläuterungen zur Variable "Steuerbelastung»}

Im theoretischen Teil wurde ausgeführt, dass Reichtum, Immobilienpreise, Steuersubstrat und Steuerbelastung einer Gemeinde eng zusammenhängen. In der folgenden empirischen Analyse wird die Steuerbelastung als Indikator für diese Merkmale benutzt. ${ }^{6}$ Bei Gemeinden mit mehr als 2000 Einwohnern kann die Steuerbelastung für die verschiedenen Einkommensgruppen einer veröffentlichten Tabelle der Eidgenössischen Steuerverwaltung (ESTV) entnommen werden. Für die kleineren Gemeinden musste die Steuerbelastung jedoch separat berechnet werden. Dazu wurde der sogenannte Steuerrechner der Bank Credit Suisse benutzt, der im Internet verfügbar ist. ${ }^{7}$ 
Tabelle 1: Variablendefinitionen

\begin{tabular}{|c|c|c|}
\hline Unabhängige Variablen & Erläuterung & $\begin{array}{l}\text { Mittelwert/Standard- } \\
\text { abweichungl }\end{array}$ \\
\hline Ausländische Bevölkerung & $\begin{array}{l}\text { Anteil an Einwohnern ausländischer Nationa- } \\
\text { lität in Prozent der gesamten Wohnbevölke- } \\
\text { rung; Min: } 1.51 \text { Max: } 46.98\end{array}$ & $19.915 / 11.201$ \\
\hline $\begin{array}{l}\text { "High-Society»- } \\
\text { Wohnatmosphäre }\end{array}$ & $\begin{array}{l}\text { Anzahl Personen, die in Einfamilienhäusern } \\
\text { wohnen, in Prozent aller Einwohner; } \\
\text { Min: 1.04 Max: } 62.21\end{array}$ & $31.460 / 16.403$ \\
\hline $\begin{array}{l}\text { Sozio-ökonomisch } \\
\text { privilegierte Personen }\end{array}$ & $\begin{array}{l}\text { Anteil an Führungskräften in der Privatwirt- } \\
\text { schaft, leitenden Verwaltungs-bediensteten } \\
\text { und Angehörigen gesetzgebender Körper- } \\
\text { schaften (höchste Kategorie der beruflichen } \\
\text { Tätigkeiten nach ISCO }{ }^{2} \text { ), in Prozent der er- } \\
\text { werbsfähigen Bevölkerung; } \\
\text { Min: } 1.97 \text { Max: } 11.17\end{array}$ & $4.854 / 1.655$ \\
\hline $\begin{array}{l}\text { Sozio-ökonomisch } \\
\text { benachteiligte Personen }\end{array}$ & $\begin{array}{l}\text { Anteil an Hilfsarbeitskräften (niedrigste Kate- } \\
\text { gorie der beruflichen Tätigkeiten nach } \\
\text { ISCO), in Prozent der erwerbsfähigen } \\
\text { Bevölkerung; Min: } 0.63 \text { Max: } 4.27\end{array}$ & $1.805 / 0.491$ \\
\hline Steuerbelastung & $\begin{array}{l}\text { Belastung durch Kantons-, Gemeinde- und } \\
\text { Kirchensteuern für einen nicht selbständig er- } \\
\text { werbenden, verheirateten Mann mit zwei Kin- } \\
\text { dern in Prozenten des Bruttojahres-einkom- } \\
\text { mens; ungewichteter, gemittelter Index aus } \\
\text { drei Einkommensgruppen, standardisiert auf } \\
\text { Mittelwert=100; Min: 44.31 Max: } 130.90\end{array}$ & $100.000 / 17.129$ \\
\hline Arbeitsmarktstruktur: & $\begin{array}{l}\text { Anzahl Beschäftigte in ausgewählten Wirt- } \\
\text { schaftszweigen }\left(\mathrm{NOGA}^{3}\right) \text { in Prozent der Bes- } \\
\text { chäftigten in Sektoren } 2 \text { und 3: }\end{array}$ & \\
\hline Textilgewerbe & Min: 0.00 Max: 16.77 & $0.817 / 2.494$ \\
\hline Chemische Industrie & Min: 0.00 Max: 11.80 & $1.018 / 2.155$ \\
\hline Metallindustrie & Min: 0.00 Max: 39.87 & $4.478 / 7.236$ \\
\hline Maschinenindustrie & Min: 0.00 Max: 57.91 & $4.078 / 6.514$ \\
\hline Baugewerbe & Min: 2.66 Max: 56.42 & $11.184 / 7.368$ \\
\hline Detailhandel/Reparatur & Min: 0.00 Max: 35.92 & $8.929 / 5.714$ \\
\hline Gastgewerbe & Min: 1.24 Max: 36.45 & $6.694 / 5.586$ \\
\hline Kreditgew./Versicherungen & Min: 0.00 Max: 21.47 & $2.144 / 2.976$ \\
\hline $\begin{array}{l}\text { Öff. Verwaltung/ } \\
\text { Landesverteidigung }\end{array}$ & Min: 0.30 Max: 17.26 & $2.808 / 2.661$ \\
\hline Unterrichtswesen & Min: 0.55 Max: 53.50 & $7.223 / 6.730$ \\
\hline Gesundheit/Soziales & Min: 0.00 Max: 37.21 & $8.836 / 7.770$ \\
\hline
\end{tabular}

1 Diese Angaben gelten für die Stichprobe von 148 Gemeinden.

2 Die ISCO (International Standard Classification of Occupations) der International Labour Organization umfasst insgesamt zehn Kategorien der beruflichen Tätigkeiten. Die erste Kategorie, welche für die Variable «sozio-ökonomisch privilegierte Personen» relevant ist, lautet in englischer Sprache «Legislators, senior officials and managers» (BFS, 2002).

3 In der "Allgemeinen Systematik der Wirtschaftszweige NOGA» werden fünf Stufen unterschieden. 
Erläuterungen zur Variable «High-Society-Wohnatmosphäre»: Die Information zur Wohnatmosphäre soll einen Eindruck davon vermitteln, ob die Wohnstruktur einer Gemeinde sozio-ökonomisch privilegierte Eltern anzieht. Es wird davon ausgegangen, dass sich letztere in Gemeinden mit vielen Einfamilienhäusern wohl und heimisch fühlen, nicht jedoch in Gemeinden, deren Ortsbild vorwiegend von Wohnblöcken und Mehrfamilienhäusern geprägt ist. Die Atmosphäre einer Gemeinde wird hier also an der baulichen Struktur festgemacht. Es wird angenommen, dass diese auch die Wohnumgebung, wie zum Beispiel die Grösse der Gärten beeinflusst. Bei dieser Variable muss jedoch auf ein Problem hingewiesen werden: Die Wohnatmosphäre kann auch innerhalb einer Gemeinde uneinheitlich sein. In den meisten Gemeinden sind Quartiere zu finden, in denen nur Wohnblöcke stehen, aber auch solche, die von Einfamilienhäusern geprägt sind. Die Wohnatmosphäre ist also strenggenommen ein sogenannter Faktor der Mikrolage. Es kann jedoch beobachtet werden, dass in gewissen Gemeinden besonders viele oder besonders wenige Personen in Einfamilienhäusern wohnen. Deshalb wird angenommen, dass es durchaus Gemeinden gibt, die auf sozio-ökonomisch privilegierte Personen besonders attraktiv wirken, da sie eben eine «High-Society»-Wohnatmosphäre ausstrahlen.

Erläuterungen zu den Variablen der «Arbeitsmarktstruktur»: Um ein Bild von der Arbeitsmarktstruktur der Gemeinden zu erhalten, wurden Wirtschaftszweige ausgewählt, in denen deutlich unterschiedliche Lohnverhältnisse herrschen. Die Information darüber, wie viele Beschäftigte verschiedener Wirtschaftszweige in einer Gemeinde arbeiten, soll in der Analyse in Beziehung gesetzt werden mit dem Anteil an sozio-ökonomisch privilegierten Personen in der entsprechenden Gemeinde. Es muss jedoch erwartet werden, dass diese Vorgehensweise für die Beantwortung der Frage nach dem Effekt des Arbeitsweges auf den Wohnortsentscheid zu kurz greift. Denn wie bereits erwähnt, wird der Arbeitsweg heute in den meisten Fällen nicht zu Fuss zurückgelegt; es ist also nicht die Frage wichtig, ob sich der Arbeitsplatz in derselben Gemeinde befindet. Wer mit öffentlichen oder privaten Verkehrsmitteln zur Arbeit fährt, misst die zeitliche Distanz - und diese kann zwischen zwei Gemeinden sogar kürzer sein als ein Arbeitsweg zu Fuss innerhalb der Wohngemeinde. Die vorhandenen Variablen sind also nicht optimal geeignet für die Überprüfung spezifischer Beziehungen im Modell; trotzdem werden sie aber als Kontrollgrössen in die Analyse miteinbezogen.

Erläuterungen zur abhängigen Variable (zweite Fragestellung): Die zweite Fragestellung betrifft den Einfluss des Reichtums einer Gemeinde auf die Ausstattung eines Schulhauses. Dazu gehören zum Beispiel Bibliotheken und Labors mit ihren Einrichtungen, oder auch Computer. Leider weist die Konstruktion und Operationalisierung verschiedener Variablen, welche die Infrastruktur im Schulhaus betreffen, im PISA-Datensatz Schwächen auf. 8 Die Aus- 
stattung eines Schulhauses wird deshalb im Folgenden lediglich an einer Variable gemessen, nämlich an der Anzahl von Computern, welche den Schülerinnen und Schülern zur Verfügung stehen. Da Computer in den Schweizer Schulhäusern noch nicht zur Standardausstattung gehören und insbesondere Investitionen in zusätzliche Computer für Schülerinnen und Schüler von den Gemeinden von Fall zu Fall beschlossen werden, eignet sich diese Variable jedoch sehr gut, um den Effekt der Steuerkraft einer Gemeinde zu überprüfen.

\section{Exploration der Daten}

Bereits eine kurze Exploration der Gemeindestichprobe lässt eine soziale Differenzierung der Gemeinden erkennen. Die Gemeinden unterscheiden sich also nicht nur durch ihren räumlichen Standort, sondern auch durch verschiedene soziale Kriterien.
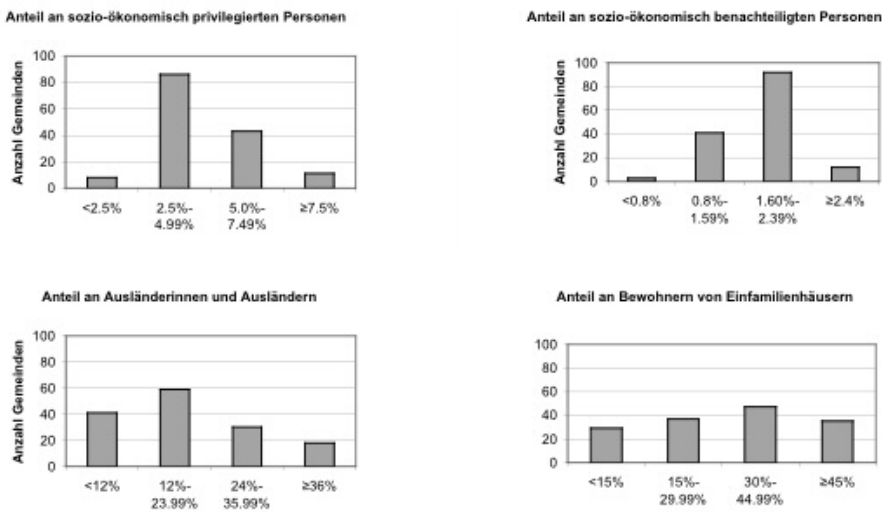

Abbildung 2: Soziale Segregation der Gemeinden

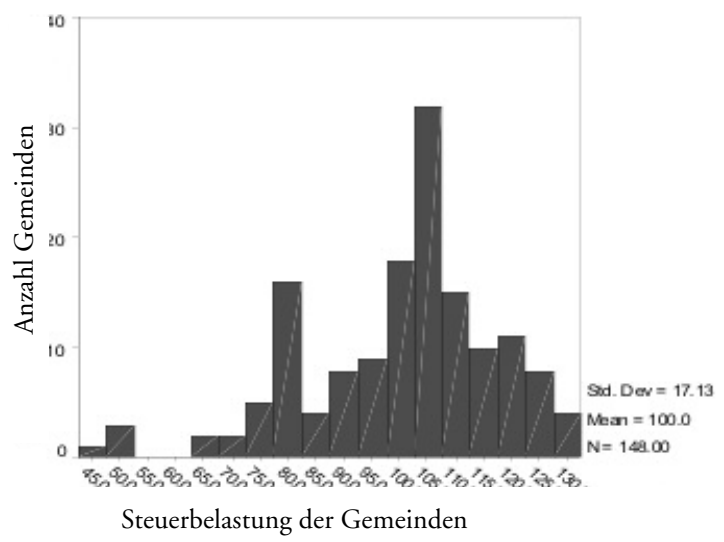

Abbildung 3: Verteilung der Steuerbelastung 
Ob aus den vorhandenen Daten gleichsam ein Profil der Differenzierungen herausgearbeitet werden kann, ist nicht von Vornherein eindeutig. Einen Einblick in die Zusammenhänge der Gemeindemerkmale kann jedoch von der folgenden multivariaten Analyse erwartet werden.

\section{Ergebnisse}

\section{Wohnort sozio-ökonomisch privilegierter Personen}

Um die Frage zu überprüfen, ob die Steuerbelastung und die Wohnatmosphäre einer Gemeinde sowie deren Arbeitsmarktstruktur mit dem Wohnortsentscheid von sozio-ökonomisch privilegierten Personen zusammenhängen, wurde eine multiple Regressionsanalyse mit den Daten der Gemeindestichprobe durchgeführt. Die folgende Tabelle zeigt die Ergebnisse.

Tabelle 2: Effekte auf den Anteil an sozio-ökonomisch privilegierten Personen in einer Gemeinde

\begin{tabular}{|l|c|c|}
\hline Merkmale & Effektgrössen & \\
\hline Konstante & Koeffizient & SE \\
Steuerbelastung & $9.436^{* * *}$ & 0.794 \\
«High-Society»-Wohnatmosphäre & $-0.052^{* * *}$ & 0.0064 \\
Textilgewerbe & $0.041^{* * *}$ & 0.008 \\
Chemische Industrie & n.s. & \\
Metallindustrie & n.s. & \\
Maschinenindustrie & $-0.028^{*}$ & 0.016 \\
Baugewerbe & n.s. & 0.016 \\
Detailhandel/Reparatur & $-0.034^{*}$ & \\
Gastgewerbe & n.s. & \\
Kreditgewerbe/Versicherungen & n.s. & \\
Öff. Verwaltung/Landesverteidigung & n.s. & 0.042 \\
Unterrichtswesen & $-0.078^{*}$ & \\
Gesundheit/Soziales & n.s. & \\
\hline $\mathrm{N}$ & n.s. & 0.50 \\
$\mathrm{R} 2$ & & \\
\hline
\end{tabular}

$*: \mathrm{p} \leq .10$

**: $\mathrm{p} \leq .05$

$* * *: \mathrm{p} \leq .001$

Mittel der abhängigen Variable: 4.854

SD der abhängigen Variable: 1.655 
Tabelle 2 zeigt, dass die Steuerbelastung signifikant negativ mit dem Anteil an sozio-ökonomisch privilegierten Personen zusammenhängt: Ist sie hoch, sind in einer Gemeinde weniger statushohe Personen zu finden. Eine Erhöhung der Steuerbelastung um 1 Prozent geht einher mit einem Rückgang des Anteils an sozio-ökonomisch privilegierten Personen um 0.052 Prozent. Das bedeutet, dass ein relativ grosser Teil der Varianz im Anteil sozio-ökonomisch privilegierter Personen durch die Steuerbelastung erklärbar ist. (Bei einer Veränderung in der Steuerbelastung um 100 Prozent resultiert eine Veränderung im Anteil an statushohen Personen von 5 Prozent. Für Gemeinden, in denen der Anteil an statushohen Personen minimal ist (1.97 Prozent), bedeutet dies: ein Rückgang der Steuerbelastung um 100 Prozent $^{9}$ ginge einher mit einer Erhöhung des Anteils an sozio-ökonomisch privilegierten Personen auf rund 7 Prozent. Das Maximum in der Stichprobe der Gemeinden liegt bei 11.17 Prozent statushoher Personen.)

Auch bei der Wohnstruktur einer Gemeinde ist ein signifikanter Effekt feststellbar: der Anteil an Bewohnerinnen und Bewohnern von Einfamilienhäusern hängt mit dem Anteil an gutsituierten Personen in einer Gemeinde zusammen. Eine Veränderung des Anteils an Bewohnern von Einfamilienhäusern um 1 Prozent geht einher mit einer Veränderung im Anteil an sozio-ökonomisch privilegierten Personen um 0.041 Prozent. Dies entspricht ebenfalls einem relativ grossen Anteil der Varianz der abhängigen Variable. Die Fragen, ob (entsprechend der theoretischen Erwartung) die Steuerbelastung den Wohnortsentscheid statushoher Personen kausal beeinflusst und die Einfamilienhäuser eine Wohnatmosphäre vermitteln, welche gutsituierte Personen anzieht, können jedoch nicht zweifelsfrei beantwortet werden. Dies wurde allerdings erwartet. Es kann davon ausgegangen werden, dass Steuerbelastung wie auch Wohnatmosphäre Ursache und Konsequenz des sozio-ökonomischen Profils einer Gemeinde sind. Eindeutig festgehalten werden kann hingegen, dass die sozial-räumliche Entmischung der Gemeinden anhand der Steuerbelastung und der Wohnatmosphäre beobachtbar ist.

Schmidheiny (2004) hat für den Agglomerationsraum Basel ähnliche Ergebnisse dokumentiert. Er stellt fest, dass reiche Haushalte mit signifikant höherer Wahrscheinlichkeit in steuergünstige Gemeinden ziehen als arme. Sozio-ökonomisch privilegierte Personen reagieren jedoch nicht nur auf Veränderungen in der Steuerbelastung von Gemeinden - die räumliche Segregation nach Einkommensgruppen muss gemäss Schmidheiny deshalb noch andere Ursachen haben. Der Autor vermutet unter Bezugnahme auf andere Studien, dass die erwartete soziale Interaktion in einer Gemeinde eine wichtige Rolle dabei spielt: reiche Personen möchten dort wohnen, wo andere reiche Personen wohnen. Das Resultat der obigen Analyse, dass sozio-ökonomisch privilegierte Personen sich auch in Gemeinden mit vielen Einfamilienhäusern gruppieren, deutet in dieselbe Richtung.

Was den Zusammenhang der Arbeitsmarktstruktur mit dem Wohnort sozioökonomisch privilegierter Personen anbelangt, können durchwegs nicht oder 
nur sehr schwach signifikante Effekte beobachtet werden. Die wenigen Effekte, die eine schwache Signifikanz aufweisen, zeichnen zudem ein etwas uneinheitliches, schwer interpretierbares Bild. Das Resultat weist darauf hin, dass die verwendeten Daten für die Analyse dieser Frage zu kurz greifen. Dies liegt unter anderem daran, dass die Nähe zum Arbeitsort nicht anhand der zeitlichen Distanz gemessen werden konnte. Die Zeit, die für den Arbeitsweg benötigt wird (und die abhängig ist von der Art, wie der Weg zurückgelegt wird), ist aber heute das wichtigste Mass für eine entsprechende Analyse. Eine andere Erklärung für die schwachen Effekte wäre, dass in jedem Wirtschaftszweig statushohe Personen arbeiten, nämlich die Führungskräfte. Ein Bezug vom Standort eines Wirtschaftszweiges und damit einer bestimmten durchschnittlichen Lohnhöhe zum Anteil an sozio-ökonomisch privilegierten Personen ist deshalb unwahrscheinlich.

\section{Materielle Ausstattung von Schulen}

Im theoretischen Teil wurde postuliert, dass die Steuerbelastung einer Gemeinde (beziehungsweise der Gemeinden im Einzugsgebiet einer bestimmten Schule) einen signifikanten Einfluss ausübt auf die Ausstattung des Schulhauses. Es wird also vermutet, dass nicht nur die Konzentration von benachteiligten Jugendlichen in einem Schulhaus negative Effekte auf ebendiese Jugendlichen ausübt, sondern auch die Tatsache, dass die Schulhäuser, welche eher von fremdsprachigen und benachteiligten Jugendlichen besucht werden, tendenziell in armen Gemeinden stehen. Umgekehrt formuliert würde dies bedeuten: statushohe Eltern, für die das Kriterium der Steuerbelastung bei der Wahl einer Wohngemeinde wichtig ist, stellen mit dieser Wahl auch gleich sicher, dass ihre Kinder in einem gut ausgestatteten Schulhaus unterrichtet werden.

In der folgenden Analyse wird untersucht, ob bestimmte Merkmale von Gemeinden, welche deren Reichtum und deren sozio-ökonomisches Profil abbilden, signifikante Effekte auf die Anzahl an Computern in den jeweiligen Schulhäusern haben. Dass qualitative Unterschiede in der Ausstattung der Schulhäuser verschiedener Gemeinden in der Schweiz durchaus möglich sind, wurde bereits dargelegt. Tabelle 3 zeigt die Ergebnisse der Analyse.

In der Tabelle ist zu sehen, dass es einen signifikanten Zusammenhang gibt zwischen der Steuerbelastung in einer Gemeinde beziehungsweise den Gemeinden im Einzugsgebiet einer Schule und der Anzahl an Computern im Schulhaus. ${ }^{10}$ In Gemeinden, in denen die Steuerbelastung hoch ist, können sich die Schulhäuser weniger Computer leisten. Oder umgekehrt formuliert: in reichen Gemeinden stehen den Schülerinnen und Schülern mehr Computer zur Verfügung als in armen Gemeinden. In vergleichbaren Grössen ausgedrückt, entspricht eine Erhöhung in der Steuerbelastung um eine Standardabweichung einem Rückgang von 34 Prozent einer Standardabweichung der Variable Anzahl Computer. 
Tabelle 3: Einflüsse auf die Anzahl an verfügbaren Computern pro Schülerin oder Schüler im Schulhaus

\begin{tabular}{|l|c|c|}
\hline Merkmale & Effektgrössen \\
Koeffizient & SE \\
Konstante & $-1.484^{*}$ & 0.713 \\
Steuerbelastung & $-0.012^{* *}$ & 0.004 \\
Sozio-ökonomisch privilegierte Personen & n.s. & \\
«High-Society»-Wohnatmosphäre & $0.013^{* *}$ & 0.005 \\
Ausländische Bevölkerung & n.s. & \\
\hline $\mathrm{N}$ & & 114 \\
$\mathrm{R}^{2}$ & & 0.15 \\
\hline
\end{tabular}

*: $\mathrm{p} \leq .10$

**: $\mathrm{p} \leq .05$

$* * *: \mathrm{p} \leq .001$

Mittel der abhängigen Variable (logarithmiert): -2.233

SD der abhängigen Variable: 0.612

Diese Ergebnisse können jedoch nicht generalisiert werden im Hinblick auf alle Gemeinden der Schweiz, sondern müssen als Tendenz verstanden werden, die sich im analysierten Datensatz von 114 Gemeinden manifestiert.

Weiter kann auch ein signifikanter Zusammenhang der Anzahl an Computern im Schulhaus mit dem Anteil an Bewohnern von Einfamilienhäusern in einer Gemeinde beobachtet werden. Eine Steigerung dieses Anteils um eine Standardabweichung geht einher mit einer Erhöhung von 35 Prozent einer Standardabweichung der Anzahl an verfügbaren Computern im Schulhaus.

Weiter oben ist gezeigt worden, dass die Steuerattraktivität und die Wohnatmosphäre einer Gemeinde signifikant mit dem Anteil an gutsituierten Personen zusammenhängen. Das Resultat in Tabelle 3 legt nun den Schluss nahe, dass solche Personen zu einem gewissen Grad zurecht annehmen, eine Schule in einer reichen Gemeinde könne ihren Kindern auch optimale Lernvoraussetzungen bieten. Kinder statushoher Eltern, die in einer reichen Gemeinde (der analysierten Stichprobe) wohnen, sind also nicht nur durch genügend finanzielle Mittel und ein förderliches Sozialisationsklima in ihrer Familie privilegiert, sondern zusätzlich durch den Besuch einer Schule, die beste materielle Lernvoraussetzungen bietet (zumindest wenn es um Computer geht). Sozio-ökonomisch benachteiligte und fremdsprachige Jugendliche hingegen, welche mehrheitlich nicht in den reichen Gemeinden wohnen, können sogar dreifach im Nachteil sein: indem ihre familiäre Lebenswelt keine optimale Voraussetzung für den Schulerfolg ist, indem sie unter Kumulationen von ebenfalls fremdsprachigen Jugendlichen in ihrem Schulhaus leiden und indem sie Schulen besuchen, die von den Gemeinden in ihrem Einzugsgebiet weniger finanzielle Mittel für die Ausstattung (z.B. mit Computern) erhalten. Diese Aussagen gelten allerdings 
nur für Jugendliche, welche Schulen mit Grundansprüchen und mit erweiterten Ansprüchen besuchen.

Nun stellt sich jedoch die Frage, ob es tatsächlich ein Nachteil für den Bildungserfolg bedeutet, wenn Jugendlichen in einer Schule vergleichbar weniger Computer zur Verfügung stehen als solchen in einer anderen Schule. Effekte dieser Variable auf die Leseleistung konnten keine nachgewiesen werden (Coradi Vellacott, in Vorb.); allerdings wurde nicht die Nutzung der Computer auf ihren Einfluss hin geprüft, sondern nur das Vorhandensein von Computern. Beim Effekt der Nutzung von Computern muss unterschieden werden, ob er im Zusammenhang mit allgemeinen schulischen Kompetenzen, wie zum Beispiel der Leseleistung diagnostiziert werden kann, oder im Zusammenhang mit der Fähigkeit, mit Computer- und Kommunikationstechnologie umzugehen. Gemäss verschiedener Forschungsarbeiten kann nur dann ein Einfluss auf die allgemeinen fachlichen Leistungen festgestellt werden, wenn der Computer in moderatem Umfang im Unterricht eingesetzt wird (Fuchs \& Wössmann, 2004). Ob ein vernünftiger Einsatz von Computern im Unterricht durch einen Mangel an Geräten in der Schule erschwert wird, kann an dieser Stelle nicht beurteilt werden. Wenn es um die Fähigkeit geht, mit Computer und Internet zu arbeiten, spielt der Ort der Nutzung, also, ob mit dem Computer in der Schule oder zu Hause gearbeitet wird, insbesondere für die Problematik der Chancengleichheit eine nicht zu unterschätzende Rolle. Ramseier und Holzer (2005) haben festgestellt, dass Jugendliche mit benachteiligter sozialer Herkunft den Computer in der Schule intensiver nutzen und diese Möglichkeit als wichtiger einstufen als solche mit privilegierender sozialer Herkunft. ${ }^{11}$ Für letztere ist hingegen die Computernutzung zu Hause wichtiger. Dies zeigt, so die Autoren, dass die Schule in gewissem Masse Rückstände in der Vertrautheit mit Computern ausgleicht. Dass nun gerade diejenigen Gemeinden, in denen viele statushohe Jugendliche wohnen, ihre Schulhäuser mit mehr Computern ausstatten als die anderen, macht vor diesem Hintergrund wenig Sinn. Wenn sozial benachteiligte Jugendliche die Computer in der Schule intensiver nutzen, jedoch weniger davon zur Verfügung haben, könnte daraus auch ein nachteiliger Effekt auf ihren Bildungserfolg resultieren, denn: für die zukünftigen Chancen auf dem Arbeitsmarkt sind nicht nur die fachlichen Leistungen, sondern auch die sogenannten Computerskills wichtig.

\section{Zusammenfassung}

In der vorliegenden Analyse wurde der Prozess der sozial-räumlichen Differenzierung in der Schweiz theoretisch und empirisch beleuchtet. Anhand einer Auswahl von Schweizer Gemeinden konnte gezeigt werden, dass sich diese in Bezug auf demographische sowie steuertechnische Merkmale erheblich unterscheiden. Zudem wurde ein signifikanter Zusammenhang zwischen dem Anteil an sozio- 
ökonomisch privilegierten Personen und der Steuerbelastung sowie der Wohnstruktur in den Gemeinden nachgewiesen. In einer systematischen theoretischen Herleitung wurde argumentiert, dass die Wohnortswahl statushoher Personen, durch die diese sozial-räumliche Entmischung zustande kommt, nicht nur von Steuern und Immobilienpreisen, sondern auch von damit verbundenen Informationen über die Qualität der jeweiligen Schule beeinflusst wird. Weiter wurde anhand eines erweiterten PISA-Datensatzes festgestellt, dass Gemeinden mit einer vergleichsweise hohen Steuerbelastung ihre Schulen mit weniger Computern ausstatten als reiche Gemeinden. In Anbetracht des Befundes, dass die soziale Zusammensetzung der Schülerschaft insbesondere für die Leistung der fremdsprachigen und benachteiligten Schülerinnen und Schüler bedeutsam ist, ergibt sich das Bild einer mehrfachen Benachteiligung spezifischer sozialer Gruppen. So sind fremdsprachige Jugendliche aus sozio-ökonomisch benachteiligten Familien, die nicht in reichen Gemeinden oder Gemeinden mit «High-Society»Wohnatmosphäre wohnen, von drei verschiedenen Nachteilen betroffen: ihre familiäre Lebenswelt ist keine optimale Voraussetzung für den Schulerfolg, sie gehen mit vielen peers zur Schule, die ebenfalls fremdsprachig sind, und sie besuchen Schulen, die von den Gemeinden in ihrem Einzugsgebiet weniger finanzielle Mittel für die Ausstattung erhalten.

\section{Anmerkungen}

1 Seit den 90er Jahren sind vermehrt Konzentrationen von Immigrantinnen und Immigranten desselben Herkunftslandes in einzelnen Häuserblöcken und kleinen Quartieren zu beobachten (Keller, 2003). Von ethnisch homogenen Ghettos kann aber noch nicht gesprochen werden.

2 Aus diesem Grund verspricht man sich in der Schweiz auch wenig positive Auswirkungen ethnischer Konzentrationen durch die Bildung tragfähiger sozialer Netzwerke (vgl. Arend, 2004). Das in soziologischer Literatur diskutierte Argument (z.B. Dear, 2000), dass im Ghetto ein Gemeinschaftsgefühl und eine Parallel-Kultur entstehen könnte, aus der heraus die Benachteiligten mit der vorherrschenden Kultur in Kontakt treten können, ist für «Sammelbecken» der Armen schwer nachvollziehbar. Arend (2004) hält in diesem Sinne fest, dass es für die normative Beurteilung der "Wünschbarkeit» von Segregation einen Unterschied macht, ob Angehörige einer ethnischen Gruppe in einem Stadtteil freiwillig nebeneinander wohnen oder ob ihre Konzentration beziehungsweise Segregation durch Armut, Diskriminierung und fehlenden Zugang zum Wohnungsmarkt erzwungen ist.

3 Wenn solche Beschlüsse Schulen der Sekundarstufe I betreffen, können sich jeweils diejenigen Gemeinden daran beteiligen, welche Schülerinnen oder Schüler in die betreffende Schule schicken, denn die Wohngemeinden kommen für die Kosten der Schülerinnen und Schüler auf.

4 An dieser Stelle müssen noch drei Bemerkungen gemacht werden. Erstens muss hier erwähnt werden, dass der Prozess der Wohnortswahl in zwei Phasen unterteilt werden kann: in den Entscheid darüber, ob abgewandert werden soll oder nicht und den allenfalls daran anschliessenden Entscheid, wohin abzuwandern sei (Kesselring, 1979). Nach Kesselring kann angenommen werden, dass für den Abwanderungsentscheid andere Gründe als Steuerbelastungs- und Versorgungsniveauunterschiede das entscheidende Gewicht haben, dass diese Unterschiede jedoch für die zweite Phase des Entscheidungsprozesses mit steigendem Einkommen immer mehr an Bedeutung gewinnen. Wie es bereits in den vo- 
rangegangenen Ausführungen deutlich gemacht wurde, geht es bei der hier behandelten Fragestellung um diese zweite Phase. Zweitens soll an dieser Stelle betont werden, dass es hier nicht darum geht, den Entscheidungsfindungsprozess selbst (zum Beispiel in einer Fallstudie über eine umzugswillige Familie) zu beobachten, sondern darum, das Ergebnis der Wohnortswahl abzubilden. Drittens ist aus demselben Grund nicht feststellbar, ob die Gemeinde, aus der weggezogen wird, die Umzugsmöglichkeiten beeinflusst (Pfadabhängigkeit).

5 Die Variablen solcher Schulen mit sehr grossem Einzugsgebiet sind nicht operationalisierbar. Gleichzeitig muss auch angenommen werden, dass sich bei solchen Schulen eine Durchmischung von unter Umständen sehr unterschiedlichen sozio-ökonomischen Profilen von Gemeinden ergibt - die zu testenden Hypothesen beziehen sich also sinnvollerweise nur auf Schulen mit limitiertem Einzugsgebiet.

6 Informationen über öffentliche Finanzen (Einnahmen und Ausgaben) werden für Gemeinden unter 10000 Einwohnern nicht zentral erfasst; es können deshalb keine solchen Angaben in die vorliegende Analyse einfliessen.

7 Um Verzerrungen durch zwei unterschiedliche Berechnungsarten zu vermeiden, wurden die rechnerischen Voraussetzungen verglichen und Kriterien eruiert, anhand derer für Beispiels-Gemeinden im Steuerrechner dieselbe Steuerbelastung resultiert wie sie von der ESTV angegeben wird.

8 Diese resultieren hauptsächlich aus einer Frageformulierung, welche für schweizerische Verhältnisse wenig Sinn macht.

9 Es geht hier lediglich um eine Verdeutlichung der Grösse des erklärten Varianzanteils, nicht um ein realistisches Beispiel. Ein Rückgang der Steuerbelastung um 100 Prozent würde bedeuten, dass in der betreffenden Gemeinde keine Steuern mehr bezahlt werden müssten.

10 Dies gilt für die Schulen mit erweiterten Ansprüchen und mit Grundansprüchen.

11 Betz (2006) stellt ausserdem mit Daten aus Deutschland fest, dass das Vertrautwerden mit dem Computer auch für Primarschulkinder mit Migrationshintergrund häufig nur in der Schule selbst erfolgen kann.

\section{Literatur}

Arend, M., Kellerhals Spitz, A. \& Mächler, T. (1990). Benachteiligte Gruppen auf dem Wohnungsmarkt. Probleme und Massnahmen. Bern: Schriftenreihe Wohnungswesen.

Arend, M. (2004). Können Stadtplanung und Wohnungsmarktpolitik einen Beitrag zur besseren Durchmischung und zur Integration von MigrantInnen leisten? In H.-R Wicker, R. Fibbi \& W. Haug (Hrsg.), Migration und die Schweiz, (S. 237-255). Zürich: Seismo Verlag.

Bayer, P., Ferreira F. V. \& McMillan, R. (2004). Tiebout Sorting, Social Multipliers and the Demand for School Quality. NBER Working Paper.

BFS Bundesamt für Statistik (2002). Statistisches Jahrbuch der Schweiz 2002. Zürich: Verlag Neue Zürcher Zeitung.

Bourdieu, P. (1985). Sozialer Raum und "Klassen»: Leçon sur la leçon, Frankfurt a. M.: Suhrkamp.

Bourdieu, P. (1991). Physischer, sozialer und angeeigneter physischer Raum. In M. Wentz (Hrsg.), Stadt-Räume. Frankfurt a. M.: Campus Verlag.

Castells, M. (1976). Is there an urban sociology? In C.G. Pickvance (Hrsg.), Urban Sociology: Critical Essays. London: Tavistock.

Coradi Vellacott, M., Hollenweger, J., Nicolet, M. \& Wolter, S.C. (2003). Soziale Integration und Leistungsförderung. Thematischer Bericht der Erhebung PISA 2000. Bundesamt für Statistik (BFS), Schweizerische Konferenz der kantonalen Erziehungsdirektoren (EDK). Neuchâtel: BFS. 
Coradi Vellacott, M. (in Vorb.). Bildungschancen Jugendlicher in der Schweiz. Eine Untersuchung familiärer, schulischer und sozial-räumlicher Einflüsse auf Leistungsunterschiede am Ende der obligatorischen Schulzeit.

Dear, M.J. (2000). The postmodern urban condition. Oxford: Blackwell.

Dear, M. J. \& Wolch, J. R. (1991). Wie das Territorium gesellschaftliche Zusammenhänge strukturiert. In M. Wentz (Hrsg.), Stadt-Räume. Frankfurt a. M.: Campus.

Ditton, H. \& Krüsken, J. (2006). Sozialer Kontext und schulische Leistungen - zur Bildungsrelevanz segregierter Armut. Zeitschrift für Soziologie der Erziehung und Sozialisation, 26, 2, 135-157.

Friedrichs, J. (1977). Stadtanalyse. Soziale und räumliche Organisation der Gesellschaft. Reinbek: Rowohlt.

Fuchs, T. \& Wössmann, L. (2004). Computers and Student Learning: Bivariate and Multivariate Evidence on the Availability and Use of Computers at Home and at School. München: Ifo and CESifo.

Giddens, A. (1987). The Role of Space in the Constitution of Society, In D. Steiner, C. Jaeger \& P. Walther (Hrsg.), Jenseits der mechanistischen Kosmologie - Neue Horizonte für die Geographie? Zürich: Geographisches Institut ETH.

Harvey, D. (1973). Social Justice and the City. London: Arnold.

Keller, R. (2003). Wohnen und Integration aus der Sicht eines Investors. Vortrag anlässlich der Grenchner Wohntage 2003.

Kesselring, H.-Ch. (1979). Kommunaler Finanzausgleich und Regionalpolitik. Grundlagen und Systematik. Eine empirische Untersuchung am Beispiel des Kantons Zürich. Zürich: Verlag Rüegger.

Leventhal, T. \& Brooks-Gunn, J. (2000). The Neighborhoods They Live in: The Effects of Neighborhood Residence on Child and Adolescent Outcomes. Psychological Bulletin, 126, 2, 309-337.

Löw, M. (2001). Raumsoziologie. Frankfurt a. M.: Suhrkamp.

Moser, U. und H. Rhyn (1997). Evaluation der Sekundarstufe I im Kanton Zürich. Zweiter Bericht. Bedingungen des Lernerfolgs. Bern: Institut für Pädagogik der Universität Bern.

Moser, U. \& Rhyn, H. (2000). Lernerfolg in der Primarschule, Aarau: Sauerländer.

Moser, U. \& Berweger, S. (2004). Einflüsse des Bildungssystems und der Schulen auf die Mathematikleistung. In Bundesamt für Statistik (BFS), Schweizerische Konferenz der kantonalen Erziehungsdirektoren ((EDK) (Hrsg.), PISA 2003: Kompetenzen für die Zukunft. Erster nationaler Bericht, (S. 45-59). Neuchâtel: BFS.

OECD Organisation für wirtschaftliche Zusammenarbeit und Entwicklung (1994). School: a Matter of Choice. Paris: OECD.

Ramseier, E. \& Brühwiler, C. (2003). Herkunft, Leistung und Bildungschancen im gegliederten Bildungssystem: vertiefte PISA-Analyse unter Einbezug der kognitiven Grundfähigkeiten. Schweizerische Zeitschrift für Bildungswissenschaften, 25 (1), 23-56.

Ramseier, E. \& Holzer, T. (2005). Vertrautheit mit Informations- und Kommunikationstechnologien (IKT). In Bundesamt für Statistik (BFS), Schweizerische Konferenz der kantonalen Erziehungsdirektoren (EDK) (Hrsg.), PISA 2003: Kompetenzen für die Zukunft Zweiter Nationaler Bericht (S.119-130). Neuchâtel: BFS.

Schmidheiny, K. (2002). Equilibrum and Stratification with Local Income Taxation when Households Differ in both Preferences and Income. Diskussionsschrift, Volkswirtschaftliches Institut der Universität Bern.

Schmidheiny, K. (2004). Income Segregation and Local Progressive Taxation: Empirical Evidence from Switzerland. CESifo Working Paper No. 1313.

Schümer, G. (2004). Zur doppelten Benachteiligung von Schülern aus unterprivilegierten Gesellschaftsschichten im deutschen Schulwesen. In G. Schümer, K.-J. Tillman \& M. Weiss (Hrsg.), Die Institution Schule und die Lebenswelt der Schüler. Vertiefende Analysen der PISA-2000-Daten zum Kontext von Schülerleistungen (S. 73-117). Wiesbaden: Verlag für Sozialwissenschaften. 
Stanat, P. (2006). Disparitäten im schulischen Erfolg: Forschungsstand zur Rolle des Migrationshintergrunds. Unterrichtswissenschaft. Zeitschrift für Lernforschung, 34, 2, 98-124.

Sun, Y. (1999). The contextual effects of community social capital on academic performance. Social Science Research, 28, 4, 403-426.

Thrupp, M., Lauder, H. \& Robinson, T. (2002). School composition and peer effects. International Journal of Educational Research, 37, 483-504.

Tiebout, Ch. M. (1956). A Pure Theory of Local Expenditures. Journal of Political Economy, 64, 416-424.

Willms, J. D. (Ed.). (2002). Vulnerable Children: Findings from Canada's National Longitudinal Survey of Children and Youth. Edmonton: University of Alberta Press.

Schlagworte: Schlagworte: Chancengleichheit; soziale Herkunft; Wohnortswahl; Leistung; Lernen; öffentliches Bildungswesen; Lernfähigkeit; Sekundarstufe I; (Wirksamkeit); OECD; (PISA); statistische Analyse; Gemeinde, Schweiz

\section{Du lien entre le choix du lieu de résidence et la qualité de l'école : réflexions théoriques et résultats empiriques}

\section{Résumé}

La composition sociale des classes de 9ème année en Suisse influence les performances individuelles - c'est ce qu'ont montré les analyses des données PISA en 2000 et 2003 . Se pose alors la question de savoir pourquoi il n'est pas possible de simplement éviter la concentration d'élèves désavantagés du point de vue linguistique et socio-économique dans certains établissements scolaires. Les théories de la différenciation spatio-sociale et du choix du lieu de résidence offrent un aperçu des processus de ségrégation sociale à l'oeuvre au sein des communes politiques et des quartiers d'habitation. A partir de ce cadre théorique, notre contribution examine d'un point de vue descriptif le rapport entre les caractéristiques des communes politiques (comme par exemple les charges d'imposition ou la qualité de vie) avec celles des quartiers où habitent les familles aisées. Cette contribution montre également l'influence de la richesse des communes politiques par rapport à l'équipement des établissements scolaires. Les connaissances théoriques et empiriques mettent aussi en évidence aussi clairement que le choix du lieu de résidence des personnes bénéficiant d'un statut social favorisé joue un rôle par rapport au processus d'homogénéisation des élèves dans les établissements scolaires de Suisse. De multiples désavantages en résultent, avant tout pour les jeunes allophones socialement défavorisés qui n'habitent pas dans des communes politiques financièrement aisées.

Mots clés: égalité de chances; origine sociale; choix du lieu de résidence; rendement; apprentissage; enseignement public; aptitude aux études; secondaire premier degré; OCDE; (PISA); analyse statistique; communes politiques, Suisse 


\section{II rapporto tra domicilio e prestazioni scolastiche: aspetti teorici e risultati empirici}

\section{Riassunto}

Come appare dai risultati svizzeri di PISA 2000 e 2003, la composizione sociale degli allievi di una scuola incide sulle prestazioni individuali ottenute al nono anno scolastico. Si pone pertanto la questione, come mai non sia possibile evitare più facilmente la concentrazione di allievi con svantaggi linguistici e socio-economici in determinate scuole. Un quadro del processo di segregazione sociale nei comuni e nei quartieri urbani lo propongono le teorie della differenziazione socio-spaziale e della scelta del luogo di domicilio. Su questa base teorica l'articolo analizza la relazione esistente tra le caratteristiche dei comuni, come ad es. il tasso fiscale o l'atmosfera abitativa, e il luogo di domicilio di persone benestanti. Si discute pure l'incidenza della ricchezza dei comuni sulla qualità delle infrastrutture delle scuole. Tanto gli aspetti teorici quanto quelli empirici mostrano come la scelta del domicilio delle persone di status sociale elevato sia in relazione con la distribuzione degli allievi sulle diverse scuole. Di conseguenza sono gli allievi di lingua straniera e di origine sociale svantaggiata che non abitano in comuni ricchi a dover sopportare gli svantaggi maggiori.

Parole chiave: parità delle opportunità, origine sociale, scelta del luogo di residenza, insegnamento pubblico, capacità di apprendimento, secondario II, OCDE, analisi statistiche, comuni politici, Svizzera

\section{Relationship between choice of residence and school quality: Theoretical considerations and empirical results.}

\section{Summary}

According to the analyses of the Swiss 2000 and 2003 PISA data, the composition of students in classes of ninth graders has an influence on their individual achievements. This leads to the question of why it is so difficult to avoid accumulations of students with language and socio-economic disadvantages in certain regions, and their schools. Looking at the processes of social segregation in communities or living quarters brings up theories of social differentiation and choice of residence. Following this theoretical background, relationships between characteristics of community (i.e., tax charge, or living atmosphere), and place of residence of wealthy people, are investigated on a descriptive level. The relationship between the wealth of a community and the level of equipment of their schools is also shown. The theoretical and empirical results make clear that the choice of residence of people with high socio-economic status and the process of social segregation in Swiss schools are interconnected. Multiple stress is also put on foreign-language students from socially disadvantaged milieus who do not live in wealthy communities.

Keywords: School quality, place of residence, socio-economic status, wealth of a community, segregation 